This is the peer reviewed version of the following article: [A. Rahmanudin; L. Yao; K. Sivula : Conjugation break spacers and flexible linkers as tools to engineer the properties of semiconducting polymers. POLYMER JOURNAL. 2018. DOI : 10.1038/s41428-018-0069-z], which has been published in final form at [https://www.nature.com/articles/s41428-018-0069-z ]. This article may be used for non-commercial purposes in accordance with Springer Nature Publishing Termss and Conditions for Self-Archiving.

\title{
Conjugation break spacers and flexible linkers as tools to engineer the properties of semiconducting polymers
}

Aiman Rahmanudin, Liang Yao, and Kevin Sivula*

Laboratory for Molecular Engineering of Optoelectronic Nanomaterials (LIMNO), École Polytechnique

Fédérale de Lausanne (EPFL), Station 6, 1015 Lausanne, Switzerland

Email: kevin.sivula@epfl.ch

TOC Graphic:

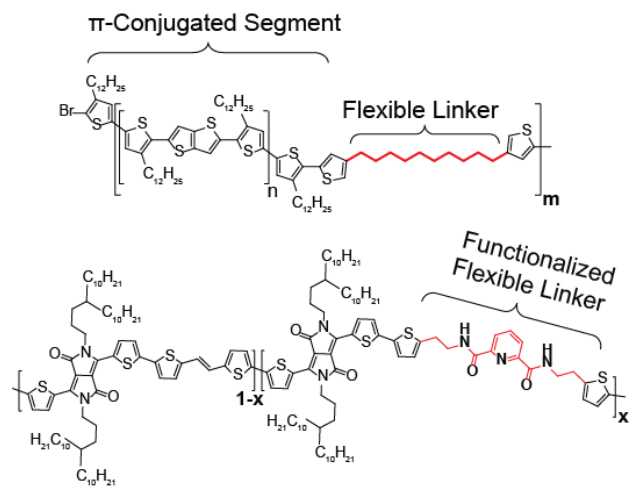

Abstract: Developing tools to understand and control the effect of semiconducting polymer morphology on the optoelectronic performance remains an important objective. Introducing conjugation break spacers (i.e flexible linkers) between $\pi$-conjugated segments in a semiconducting polymer is an emerging strategy toward this goal. Herein we place this strategy in context with other extrinsic and intrinsic engineering approaches and highlight some of the recent results employing this "flexible linker" approach. We see that the inclusion of electrically insulating aliphatic spacers represents a versatile tool to gain insight into the nature of interand intra- molecular charge carrier transport and can be broadly used to control morphology of solutionprocessed semiconducting polymer thin films. Moreover, this approach has afforded unique control over material processing and mechanical properties (e.g. viscosity and elasticity) without detrimental effect on the semiconducting ability. While the development of this technique remains at an early stage, its potential gives promise to reaching the goal of engineering the self-assembly of semiconducting polymers

Keywords - molecular engineering, organic semiconductors, solution-processing, self-assembly, morphology, photovoltaics, transistors

\section{Introduction}

Over the past four decades, $\pi$-conjugated semiconducting polymers have attracted significant attention from both academic and industrial laboratories due to a wealth of potential applications in optoelectronic devices. 
Recently, the advancement of semiconducting polymers in solution-processed thin-film organic lightemitting-diodes, ${ }^{1}$ field-effect transistors, ${ }^{2}$ photovoltaics, ${ }^{3}$ and bioelectronics ${ }^{4}$ has given genuine promise to the possibility of inexpensive roll-to-roll fabrication or inkjet printing of high performance devices using these materials. The key to progress in the field has come from innovation in synthetic methodology and material design, which has been extensively applied to manipulate the molecular structure of the $\pi$-conjugated polymer backbone and solubilizing side chains. This work has led to a vast library of oligomeric and polymeric semiconductors with tuneable functionality. ${ }^{5,6}$ However, while the control over the optoelectronic properties and processability of $\pi$-conjugated semiconducting polymers has been extensively investigated, the behaviour of polymer semiconductor thin film devices is very complex. Indeed the chains of typical solutionprocessable semiconducting polymer consist of a semi-rigid backbone and flexible (solubilizing) sidechains. In the solid state these polymer chains do not form amorphous coils nor do they self-assembly into a perfect crystalline structure. Rather the solid-state morphology is somewhere in between, and a major challenge remains in the understanding of how the molecular structure translates into a solid-state morphology and further how this morphology dictates performance. ${ }^{7,8}$

For example, a fundamental performance metric of polymer semiconductors is the solid state charge carrier mobility (e.g. hole mobility, $\mu_{h}$ ). It is well known that charge transport in semiconductor polymers in the solid state occurs through relatively fast intramolecular charge carrier migration along the $\pi$-conjugated backbone together with the intermolecular hopping of charges between conjugated segments in the $\pi$-stacking direction. While much effort has been directed toward accurately predicting $\mu_{h}$ based on density functional theory (DFT), consideration of the complex thin-film morphological structure to precisely model the relationship between intermolecular and intramolecular transport limits the precision of these predictions. ${ }^{9-}$ ${ }^{11}$ This is apparent in the disaccord between the $\mu_{\mathrm{h}}$ predicted using DFT and the experimentally measured values in a thin film transistor (TFT) device. Indeed, for the prototypical thiophene-based semiconducting polymers such as poly(3-hexylthiophene), P3HT, and poly-2,5-bis[3-alkylthiophen-2-ylthiono(3,2b)thiophene], PBTTT, the predicted $\mu_{\mathrm{h}}$ values are as high as 31 and $15 \mathrm{~cm}^{2} \mathrm{~V}^{-1} \mathrm{~s}^{-1}$, respectively, but TFT measurements give only up to 0.1 and $1.0 \mathrm{~cm}^{2} \mathrm{~V}^{-1} \mathrm{~s}^{-1}$. ${ }^{12}$ While this discrepancy indicates that optoelectronic performance is largely dictated by the thin film morphology of the polymer semiconductor, it also suggests 
that substantial improvement in performance remains attainable if the solid-state self-assembly of these materials can be controlled and understood over various length scales. ${ }^{13}$ However, defects, ${ }^{14,15}$ quasicrystallinity, ${ }^{16}$ polymorphism, ${ }^{17-19}$ domain orientation/grain boundaries, ${ }^{20,21}$ and phase separation (in blends or mixtures) $)^{22}$ have all been identified as influential to the experimentally measured performance of solidstate semiconducting polymers and attempts in predicting these aspects and their effect on charge transport has remained a challenge. ${ }^{23-25}$ Therefore, developing a set of tools to experimentally control morphology and crystallinity in polymer semiconductors is now under development to fully rationalize links between molecular structure, self-assembly, and function in $\pi$-conjugated semiconductors. This is in effort to further advance the field towards improving device performance ${ }^{26}$ and realizing unique functionalities that open the field to novel applications. ${ }^{27-29}$ In this focus review, we will briefly overview the tools used to control selfassembly and morphology in $\pi$-conjugated polymer semiconductors and highlight results using a promising and emerging approach i.e. employing conjugation break spacers (or flexible non-conjugated linkers) to control self-assembly.

\section{Tools for controlling self-assembly in $\pi$-conjugated polymer semiconductors}

Many tools have been developed to control the solid-state morphology of semiconducting polymers by modulating their self-assembly during the casting of the materials from solution. Extrinsic morphology control techniques such as the variation of solvent/processing conditions, meniscus-guided coating, and printing ${ }^{30}$ have been established as powerful tools in controlling the thin film morphology in semiconducting polymers. Moreover the use of processing additives such as nucleation promoters, ${ }^{31}$ electronically insulating polymers, $^{32}$ and post-deposition treatments ${ }^{33}$ have also been exploited. These approaches are greatly influenced by external factors, i.e. the nature of substrate and solvent, evaporation rate, and processing technique used. Thus extensive empirical optimization is required and the results are often not universal towards arbitrary molecular structure. ${ }^{13}$ On the other hand, molecular engineering approaches can directly impact the intrinsic self-assembly of the $\pi$-conjugated semiconductor. ${ }^{34}$ For instance, modulating the $\pi$ conjugated backbone by introducing highly planar $\pi$-extended conjugated monomer units, ${ }^{35,36}$ alternating donor-acceptor moieties, ${ }^{37}$ restricting rotation between monomeric units via covalent bonds (i.e. ladder-type polymers) $)^{38}$ or using non-covalent conformational locks, ${ }^{39}$ have all shown the ability to control the observed 
solid-state conjugation length and $\pi-\pi$ stacking interactions, which directly affect the intra- and intermolecular charge transport, respectively (although we note that in some cases, addition of planar comonomer units results in a curved backbone structure that hinders intermolecular electronic communication). ${ }^{40}$ Alternatively, side-chain engineering, which is typically used to induce solubility, can also be employed to modulate $\pi-\pi$ stacking of neighbouring conjugated segments.$^{41}$ Linear alkyl chains minimize intermolecular steric hindrances leading to 1D co-stacking of the conjugated backbone, while branching distorts efficient stacking. ${ }^{42,43}$ Interestingly, shifting the position of the branching point away from the conjugate core induces extremely short $\pi-\pi$ stacking distances without comprising solubility. ${ }^{44}$ In addition, inducing ordering by hydrogen bonding of urea containing groups, ${ }^{45}$ or functionalizing side chains via fluorinated alkyl chains with strong self-organization, ${ }^{46}$ have shown to drive a high degree of order between $\pi$-conjugated segments. Despite the potential of side-chain engineering in affecting self-assembly, it should be noted that $\pi-\pi$ stacking interactions still dominate the overall supramolecular assembly. ${ }^{47}$

Recently, a method to control the supramolecular assembly without altering the side chains or changing the energetics of the $\pi$-conjugated core has emerged for semiconducting polymers in OFET and OPV applications. This strategy consists of covalently joining $\pi$-conjugated segments with a flexible non-semiconducting spacer - typically an aliphatic chain (See Figure 1a). ${ }^{48-51}$ Since the non-conjugated spacer interrupts the continuous conjugation typical of most semiconducting polymers, the spacers are often referred to as "conjugationbreak spacers." Alternatively, since the spacers also add a degree of conformational freedom to the otherwise rigid conjugated backbone, they have also been referred to as "flexible linkers." Intuitively, the inclusion of non-conjugated linkers along the polymer backbone could be expected to disrupt intramolecular charge carrier transport and create a high degree disorder in the material in the solid state. While at first glance this may be thought to only negatively impact the optoelectronic properties, recent demonstrations have highlighted the usefulness of this approach in easing backbone rigidity to enhance processability, and offering unique self-assembly motifs for efficient device performance..$^{52-61}$ In the next sections we describe the historical development and specific applications of this flexible linker approach. 


\section{Initial demonstrations of conjugation-break spacers with poly-phenylenevinylene}

The concept of linking rigid conjugated segments with flexible spacers is not new and was initially developed in the 1970 s and 80 s in the field of liquid crystalline polymers. ${ }^{62}$ Specifically, thermotropic "main-chain" liquid crystalline polymers were extensively studied, and found to exhibit a wide variety of unusual mechanical and rheological phenomena. ${ }^{63}$ However, the optoelectronic properties of this class of materials was not widely considered until semiconducting poly-phenylenevinylene (PPV) polymers became of broad interest. The concept of incorporating non-conjugated linkages in the backbone of PPV by copolymerization ${ }^{64}$ or by partially saturating the vinyl linkages via selective elimination ${ }^{65-67}$ was investigated in the 90 s and early 2000 s for application in OLED devices to tune the band-gap, ease rigidity of the polymer backbone and enhance solution processability. However, the effect on the charge carrier transport was generally negative. In 2011 Barbara and co-workers used the Horner method to synthesize random copolymers of poly(2-methoxy-5-(2'ethylhexyloxy)-p-phenylene vinylene) (MEH-PPV) that incorporated different backbone-directing monomers. ${ }^{68}$ Rigid and bent defects were found to lower the anisotropy of the single chain, while saturated defects provided rotational freedom for the chain backbone allow for a wide variety of possible configurations. Plunkett and co-workers followed up on this work with PPV oligomers linked via flexible alkyl and glycol chains termed "flexible morphons" to direct the extent of the interchain interactions in solid state. ${ }^{69}$ While the solution photophysical properties between the non-linked PPV oligomers and polymerincorporated PPVs were very similar, their approach provided finer control over effective conjugation length and resulting thin-film properties of the conjugated PPV polymer. ${ }^{70}$ Overall these studies, while not demonstrating practical optoelectronic device applications, laid the groundwork for being able to design semiconducting polymer morphologies whereby the addition of different directing groups to the main chain backbone could influence the molecular self-assembly of the polymer chains in the manner desired.

\section{Decoupling inter- and intra- molecular charge transport using a flexible linker approach}

In a seminal demonstration of the application of the flexible linker approach to optoelectronic device applications, Gasperini et al. gained important insight into the relationship between self-assembly and charge transport in PBTTT. ${ }^{44}$ In that work, short PBTTT segments ${ }^{71}$ were connected into a flexibly-linked structure 
coded as FL-PBTTT and shown in Figure $1 \mathrm{a}$ (with $\mathrm{n}=10-12, \mathrm{~m}=4-5$ ). It should be noted that the presence of free short PBTTT segments in the final sample was excluded by purification with preparatory size-exclusion chromatography, prep-SEC. In sold state thin films, FL-PBTTT was found to exhibit distinct morphologies from rod-like fibrils to terraces (see height topography images in Figure $1 \mathrm{~b}-\mathrm{d}$ ) by simply altering the temperature of a post-deposition thermal treatment. The crystallinity of the thin films was accordingly altered as well with the $130{ }^{\circ} \mathrm{C}$ annealing condition (Figure 1c) producing the most crystalline film (with a more random alignment of crystalline domains) compared to the as-cast or $180^{\circ} \mathrm{C}$ films (Figures $1 \mathrm{~b}, \mathrm{~d}$ ), which were found to have an alignment of the lamellar stacking of the crystal domains aligned with the substrate (as seen by the 2D grazing-incidence X-ray diffraction plots in the insets of Figure 1b-d). Importantly, this showed that without changing the length of the conjugated segments, the self-assembly could be drastically altered. In TFTs, changing the film morphology gave an improvement of the charge carrier mobility from fibril-type $\left(0.01 \mathrm{~cm}^{2}\right.$ $\left.\mathrm{V}^{-1} \mathrm{~s}^{-1}\right)$ to terrace morphologies $\left(0.04 \mathrm{~cm}^{2} \mathrm{~V}^{-1} \mathrm{~s}^{-1}\right)$, while actually decreasing the overall crystallinity of the film. These result suggest that the high $\mu_{\mathrm{h}}$ observed in medium molecular weight PBTTT (e.g. $\left.\mathrm{n}=50\right)$ without a flexible linker is not solely due to improved intramolecular transport as suggested in one report, ${ }^{72}$ but rather reinforces the notion described by Salleo and co-workers, i.e. that the 2D charge-transport network afforded by the self-assembly significantly contributes to the observed high charge carrier mobility. ${ }^{16}$ 


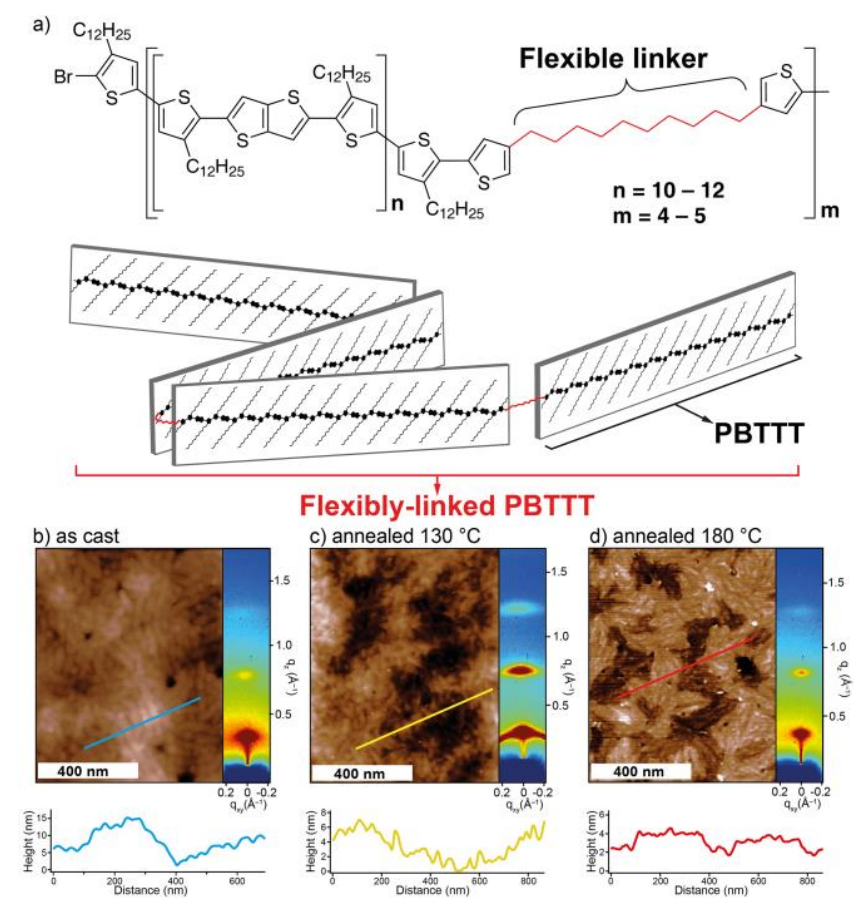

Figure 1. a) (top) chemical structure a flexibly linked polymer FL-PBTTT and (bottom) a schematic of the structure indicating the relative rigidity of the conjugated segments and their approximate length/number. Tapping mode AFM topography of: b) FL-PBTTT as cast from o-dichlorobenzene $20 \mathrm{mg} \mathrm{mL}^{-1}$, c) FL-PBTTT after annealing at $130^{\circ} \mathrm{C}$, and d) after annealing at $180^{\circ} \mathrm{C}$. The topographical profile along the indicated diagonal line in each case is shown below. The right side of each panel shows the 2D grazing -incidence X-ray diffraction plots of the same films with the vertical direction corresponding to the out-of-plane scatting vector, $q_{z}$, and the horizontal direction corresponding to $q_{x y}$. Red areas represent the highest scattering intensity while blue represent the lowest. Adapted from Ref. ${ }^{54}$ C The Royal Society of Chemistry 2014.

On the other hand, it should be noted that the recently developed polymer semiconductors based on napthalenediimides, ${ }^{73}$ diketopyrrolopyrrole, ${ }^{74}$ and carbazoles, ${ }^{75}$ do not exhibit long range crystalline order like P3HT and PBTTT but exhibit superior $\mu_{\mathrm{h}}$ over $1 \mathrm{~cm}^{2} \mathrm{~V}^{-1} \mathrm{~s}^{-1}$. Interestingly, despite a seemingly disordered morphology, these polymers exhibit aggregation consistent with improved intermolecular associations (indicative from resolvable vibronic progression near the absorption edge, and red shifting in their optical absorption spectra). ${ }^{76}$ This indicates that the short-range ordering of the aggregates is sufficient for efficient intermolecular charge transport, so long as the aggregates are sufficiently interconnected. ${ }^{77}$ Overall these observations have led to a design principle for efficient charge mobility in a conjugated polymer film, whereby rather than inducing high crystallinity, improving the interconnectedness between aggregated domains, and reducing the amount of disorder within conjugated segments (making rigid backbones) seems to be the key to facilitate the optimum?intra- and intermolecular charge transport at the device length scale. 


\section{Controlling morphology and film formation of highly crystalline semiconductors using a flexible linker}

Based on the notion that interconnectedness of domains is key to optimizing charge transport in organic semiconductors, the flexible linker strategy can also contribute useful control of this aspect. Indeed, this strategy has been employed to improve the connectivity of domains in highly crystalline solution processed thin films of small-molecule semiconductors. Despite the purported advantages of small molecule (or molecular) semiconductors over polymer semiconductors (including synthetic simplicity and the ability to remove batch-to-batch variations $)^{78}$ this class of $\pi$-conjugated semiconductors typically self-assemble into highly crystalline domains that result in film dewetting, unpredictable crystallite dimensions, and grain boundaries which confound the morphological control and charge transport in devices fabricated from these materials. To address this, a flexible linker concept was applied to a common molecular semiconductor, coded as DPP(TBFu) 2 or 3,6-bis(5-(benzofuran-2-yl)thiophen-2-yl)-2,5-bis(2-ethylhexyl)-2,5dihydropyrrolo[3,4-c]pyrrole-1,4-dione, resulting in a polymer with flexibly-linked conjugated segments identical to DPP(TBFu $)_{2}$, and coded as FL-DPP $(\mathrm{TBFu})_{2}$ (See Figure 2a)..$^{49}$ It should be noted that the synthesis of FL-DPP(TBFu $)_{2}$ was simply performed via the Stille cross coupling condensation polymerization using a functionalized bis(benzofuranyl)alkane. TFTs based on pure FL-DPP(TBFu) ${ }_{2}$ showed no measureable $\mu_{\mathrm{h}}$ (which is likely due lack of intramolecular charge transport since the $\pi$-conjugated backbone of small molecules are independent of each other). However, we note that a comparable system based on poly(rod-coil) polymers consisting of short benzothiophene centred acceptor-donor-acceptor (A-D-A) type ${ }^{51}$ and diketopyrrolopyrrole centred ${ }^{79}$ D-A-D conjugated segments linked with non-conjugated aliphatic spacers did exhibit hole transporting properties in a bulk heterojunction organic photovoltaic (OPV) device with a modest maximum device performance of c.a 1.0\%. Despite the poor charge transport of this class of materials, the FL-DPP(TBFu $)_{2}$ proved to be useful as an additive to films of DPP(TBFu $)_{2}$. Indeed, blending FL-DPP $(T B F u)_{2}$ with $\operatorname{DPP}(\mathrm{TBFu})_{2}$ exhibited interesting results when subjected to thermal stress (at $100{ }^{\circ} \mathrm{C}$ ) for $3 \mathrm{~h}$ (See Figure $2 \mathrm{~b}$ ). TFTs containing pure DPP(TBFu $)_{2}(0 \mathrm{wt} \%)$ gave $\mu_{\mathrm{h}}$ that decreased by an order of a magnitude (due to film dewetting) whereby a considerably lower decrease was observed when 1 wt $\%$ of the FL-DPP(TBFu $)_{2}$ was added, and notably at $5 \mathrm{wt} \%$, the $\mu_{\mathrm{h}}$ remained constant. This indicates the ability of the FL-DPP(TBFu ${ }_{2}$ to 
control and stabilize the crystalline domain formation in the thin film. A further demonstration of this was accomplished with bulk heterojunction blends of $\mathrm{DPP}(\mathrm{TBFu})_{2}$ with phenyl- $\mathrm{C}_{61}$-butyric acid methyl ester $\left(\mathrm{PC}_{61} \mathrm{BM}\right)$ in OPV devices. Morphological topography and young modulus mapping via atomic force microscopy (AFM) of the $\mathrm{BHJ}$ blend films under prolonged annealing (at $100^{\circ} \mathrm{C}$ ) exhibited similar intermixed $\mathrm{BHJ}$ morphology between as-cast films and after 22 hours at $100^{\circ} \mathrm{C}$ with $1.5 \mathrm{wt} \%$ of FL-DPP(TBFu $)_{2}($ See Figure $2 \mathrm{c}$, and $2 \mathrm{e}$ ) indicating a preservation of the $\mathrm{BHJ}$ morphology. On the other hand, with $0 \mathrm{wt} \%$ the $\mathrm{BHJ}$ was found to be devoid of the $\mathrm{PC}_{61} \mathrm{BM}$ as it was excluded due to the crystallization of the $\mathrm{DPP}(\mathrm{TBFu})_{2}$ (See Figure 2d). This morphological evolution correlated well with the preservation of device performance (See Figure 2f). While the control (0 wt\%) device decreased in power conversion efficiency with respect to time under thermal stress due to phase segregation, including only $0.5 \mathrm{wt} \%$ of the FL-DPP(TBFu $)_{2}$ stopped this process from occurring. Considering the polymeric structure of the FL-DPP(TBFu $)_{2}$, a plausible explanation for the observed behaviour suggests that the FL-DPP $(\mathrm{TBFu})_{2}$ acted as a tie-in molecule to bridge adjacent crystal domains of DPP(TBFu $)_{2}$, effectively locking-in the active layer morphology. 

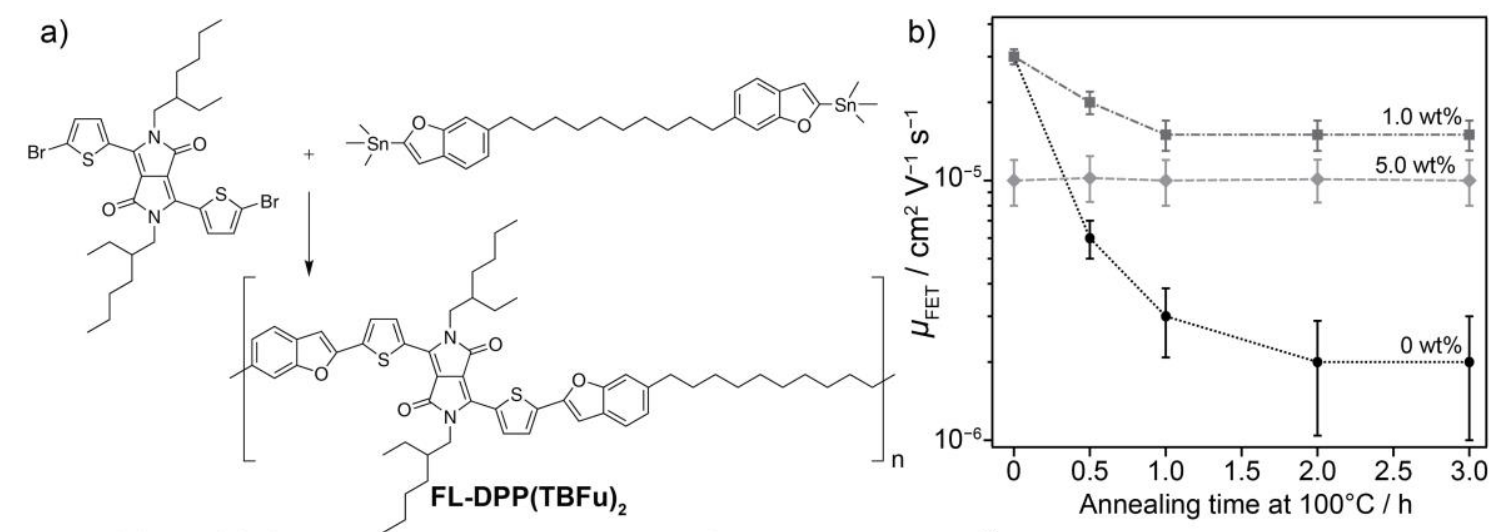

c) $0 \mathrm{wt} \%, 100^{\circ} \mathrm{C}$ for 10 minutes $\left.\left[\mathrm{DPP}(\mathrm{TBFu})_{2}: \mathrm{PC}_{61} \mathrm{BM}(3: 2)\right] \mathrm{GPa} \quad \mathrm{f}\right)$

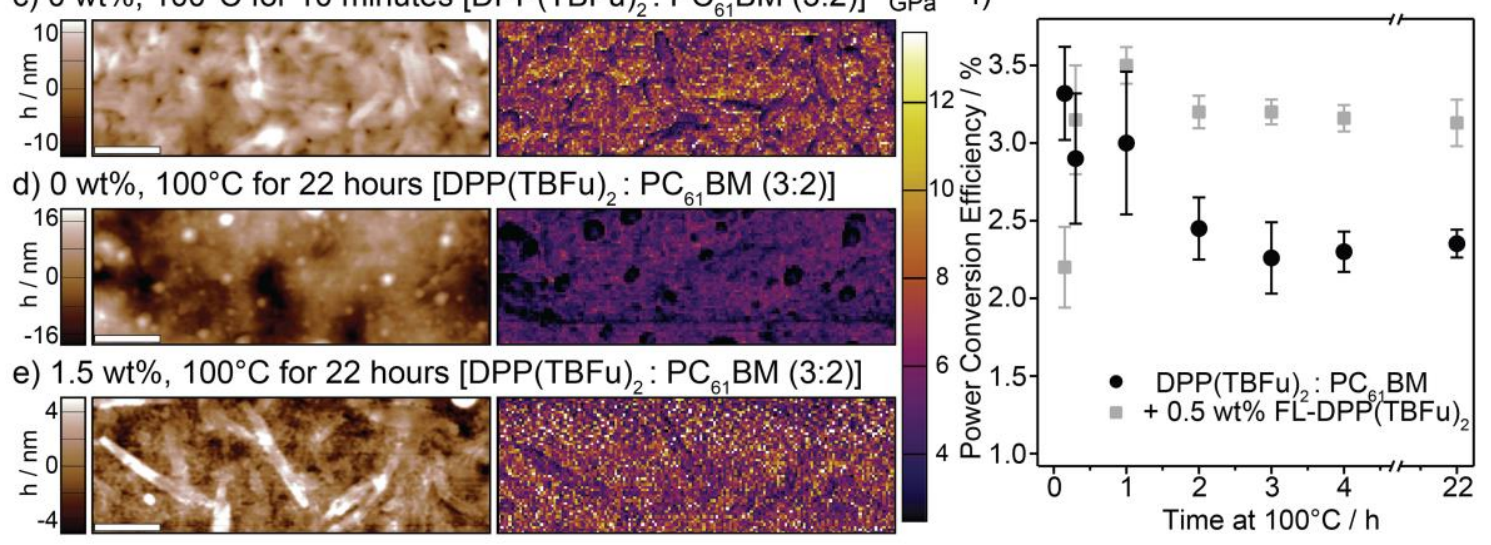

Figure 2. A flexibly-linked small molecule semiconductor. Panel (a) shows the synthetic scheme for FL-DPP(TBFu) 2 , the polymer used to control the morphology of its parent (non-flexibly linked) small-molecule semiconductor, DPP(TBFu)2. (b) shows the thin film transistor performance of blended FL-DPP(TBFu $)_{2}$ : DPP $(T B F u)_{2}$ at different wt\% of the FL polymer mobility as a function of device annealing time at $100^{\circ} \mathrm{C}$. The morphology of bulk heterojunctions of $D P P(T B F u)_{2}: P C_{61} B M$ with or without FL-DPP(TBFu) 2 is shown in panels (c-e) with topography (left side) and Young's modulus mapping (right side) of the corresponding area (the scale bars are 500nm). Panel ( $f$ ) shows the OPV PCE as a function of the active layer annealing time at $100^{\circ} \mathrm{C}$ of devices with $0 \mathrm{wt} \%$ and $0.5 \mathrm{wt} \%$ of FL-DPP(TBFu)2. Figures are adapted from Ref. ${ }^{49}$ C 2015 WILEY-VCH Verlag GmbH \& Co. KGaA.

An important general question regarding the use of the aliphatic linking technique is the effect of the linking position. To gain insight into this aspect with the model DPP(TBFu $)_{2}$ system, Jeanbourquin et al. compared two distinct dimer analogues (See structures in Figure 3)..$^{80}$ The "vertically-linked," V-(DPP) 2 , and the "horizontally-linked," $\mathrm{H}$-(DPP) 2 , dimers were both found to have the same optical band-gap of the parent $\mathrm{DPP}(\mathrm{TBFu})_{2}$ molecule, but exhibit very different self-assembly properties. Interestingly, while $\mathrm{V}$-(DPP) ${ }_{2}$ exhibited poor crystallinity in pure films, it enhanced $\mu_{\mathrm{h}}$ in FETs by 10 -fold, when used as an additive with the primary $\mathrm{DPP}(\mathrm{TBFu})_{2}$ molecule. This increase was ascribed to a nucleation promotion effect of the dimer, where fewer large charge-trapping grain boundaries were observed but the crystallinity of the DPP $(T B F u)_{2}$ in the thin film remained present. On the other hand, $\mathrm{H}-(\mathrm{DPP})_{2}$, had no significant effect in charge carrier transport, but was found to increased thermodynamic miscibility between the donor and acceptor phases 
which induced an enhanced $\mathrm{BHJ}$ thermal stability in $\mathrm{DPP}(\mathrm{TBFu})_{2}: \mathrm{PCBM}$ blends. OPV devices accordingly retained $90 \%$ of their initial conversion efficiency after 5 hours of thermal treatment (at $100^{\circ} \mathrm{C}$ ), compared to a $45 \%$ retention for the devices without the dimer additive.

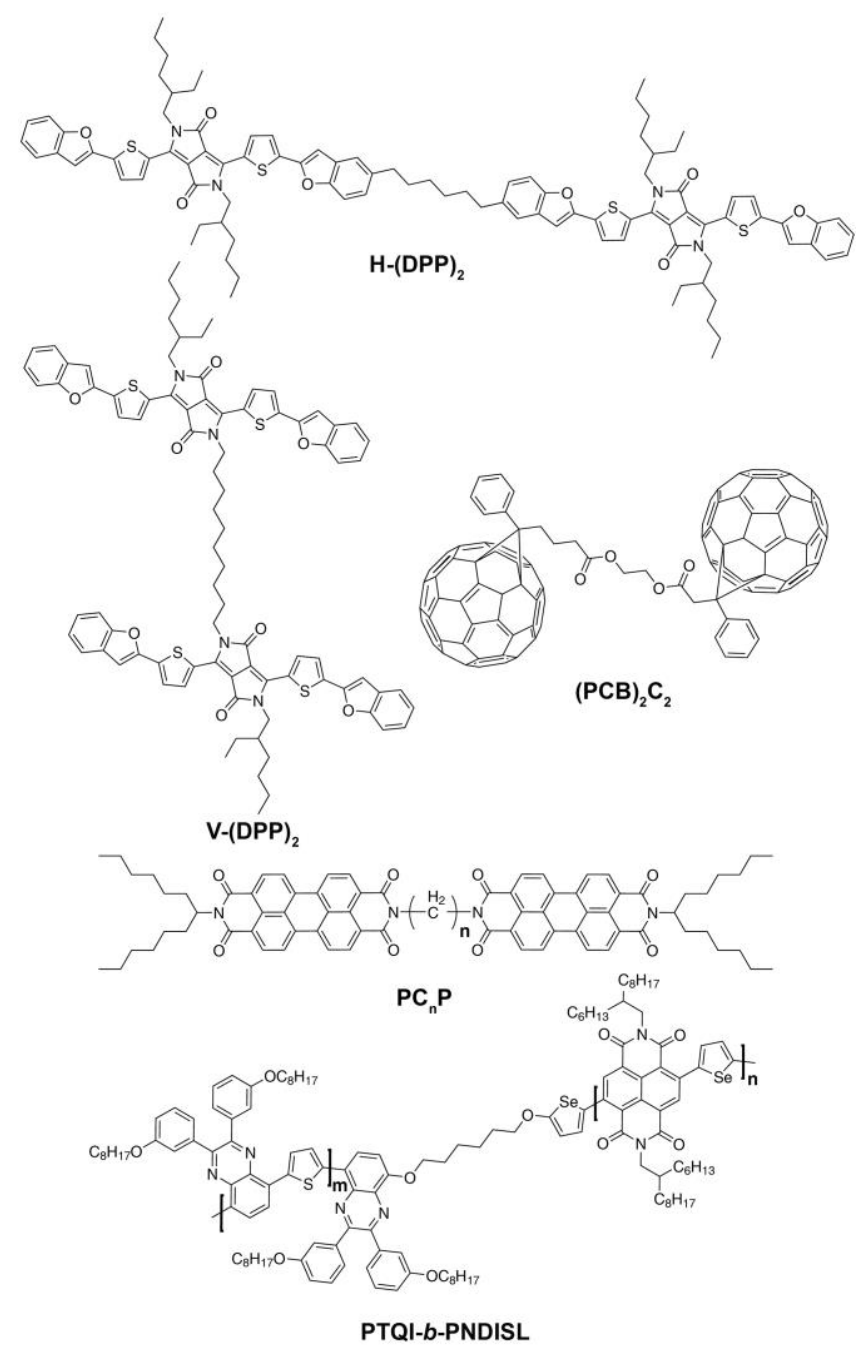

Figure 3. Chemical structures of flexibly linked small-molecule and block-co polymers discussed in the main text.

Since this dimer linking strategy is easily generalizable it has been exploited for a number of other molecules. For example, McCulloch and co-workers demonstrated a "dumbbell" fullerene dimer, $(\mathrm{PCB})_{2} \mathrm{C}_{2}$ (See Figure 3 for chemical structure) bridged by an alkyl spacer attached on the ester functional group of PCBM. ${ }^{81}$ The dumbbell dimer inhibited the formation of micron-scale crystallites of the parent PCBM molecule when used as an additive in a BHJ blend with a polymer donor, which improved OPV device lifetimes by $20 \%$ under thermal stress (at $80^{\circ} \mathrm{C}$ ) as compared to blends without the dumbbell dimer additive. A notable example by Yagai and co-workers demonstrated a dimension-controlled self-organization of perylenediimide (PDI) 
dimers coded as $\mathrm{PC}_{n} \mathrm{P}$ (See Figure 3 for chemical structure), where the relative orientation of tethered PDI units is regulated by the odd/even effect of the flexible linkers which influence its packing structures. ${ }^{82}$

In addition to dimers, the flexible linker approach was also recently utilized in a donor-b-acceptor blockcopolymer (BCP) system, ${ }^{83}$ where nanophase segregation of the respective donor and acceptor block remains a pertinent challenge. ${ }^{84,85}$ Introduction of a non-conjugated alkyl spacer between the donor and acceptor blocks of the BCP to give the polymer coded as PTQi-b-PNDISL (see Figure 3 for structure) permitted an increase in the degrees of conformational freedom, as compared to the fully-conjugated BCP. This allowed the two electronically distinct blocks to crystalize independently forming a prominent nanophase separation behaviour where a significantly larger OPV power conversion efficiency of $1.54 \%$ was obtained as compared to $0.36 \%$ of the fully conjugated BCP. A similar approach was also employed in a small molecule BHJ based on DPP(TBFu $)_{2}: \mathrm{PCBM}$, where the donor and acceptor semiconductor were connected with an aliphatic spacer to form a molecular compatibilizing additive. ${ }^{86}$ The additive demonstrated significant control over the degree of phase segregation in the small molecule BHJ that stabilized its OPV device performance under thermal stress.

Overall, these reports demonstrate that linking conjugated segments with a flexible aliphatic chain is a promising approach to control molecular self-assembly without changing the nature of the semiconducting molecular core. Further exploitation of this strategy will likely lead to an increased understanding of the important relationships between molecular self-assembly and the performance of organic electronic devices.

\section{Tuning mechanical properties and processability with a flexible linker approach}

Another notable aspect of the flexible linker strategy is the ability to tune the processabililty of $\pi$-conjugated semiconductors. Indeed, as the rigidity of backbone increases the critical polymer length for entanglement also increases (as evidenced by comparing entanglement molecular weights for the classic polymers $\mathrm{P}^{3} \mathrm{HT}^{87}$ and $\mathrm{PBTTT}^{71}$ ). The polymer chain entanglement will not only greatly affect processability, but also, for conjugated polymers, it will influence the molecular self-assembly and resulting carrier transport. ${ }^{88}$ Bao and co-workers recently showed that the incorporation of non-conjugated flexible linkers into an isoindigo based semiconducting polymer coded Pil2T-X (see chemical structure in Figure 4a) affected the processability of 
this material through modulating its viscoelastic properties. ${ }^{56}$ Rheometry and dynamic mechanical analysis revealed that solution viscosity was directly proportional to the content of non-conjugated linkers in the polymer backbone. Indeed, flow curves (Figure 4b) indicate that the viscosity of the polymer solutions in dichlorobenzene (o-DCB) decrease from 0.1 Pa s (Pil2T control without flexible linker) to $0.01 \mathrm{~Pa} s$ when $20 \%$ of the flexible linker monomer was included in the polymer backbone (Pil2T-20). The effect of the side chains is also highlighted here as the Pil2T-Ref polymer, which has shorter 2-octyldodecyl side chains, was found to gel under the same conditions and have a remarkably higher viscosity of $1 \mathrm{~Pa}$ s. Interestingly, the resulting measured $\mu_{\mathrm{h}}$ in TFTs were unexpectedly high maximum compared to other flexibly linked polymers (e.g. FLPBTTT) reaching a carrier mobility of $1.4 \mathrm{~cm}^{2} \mathrm{~V}^{-1} \mathrm{~s}^{-1}$ with Pil2T-5 and $0.23 \mathrm{~cm}^{2} \mathrm{~V}^{-1} \mathrm{~s}^{-1}$ with Pil2T-20 despite having a non-fully conjugated polymer backbone. Due to the good semiconductor properties and the enhanced processability due to the lower viscosity, a solution shearing method used to coat large-area substrates could be employed using Pil2T-X as donor polymer in an all-polymer solar cell (with a PDI polymer acceptor). The device with Pil2T-5 or Pil2T-20 achieved reasonable power conversion efficiency values of 3.7 or $3.0 \%$, respectively.

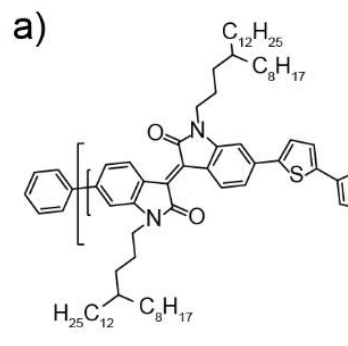

c)

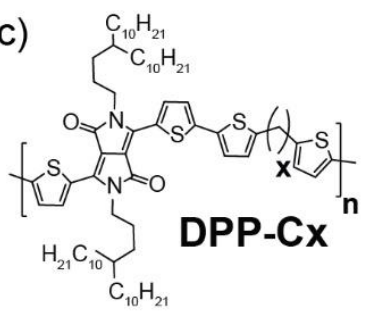

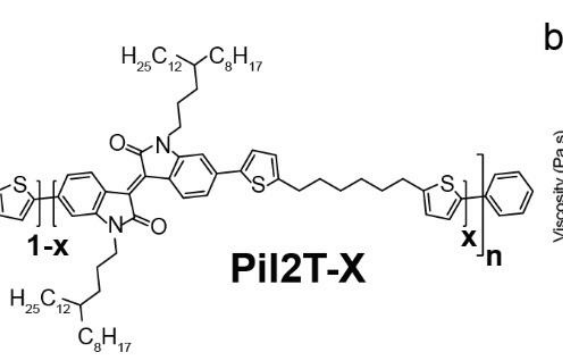

d)

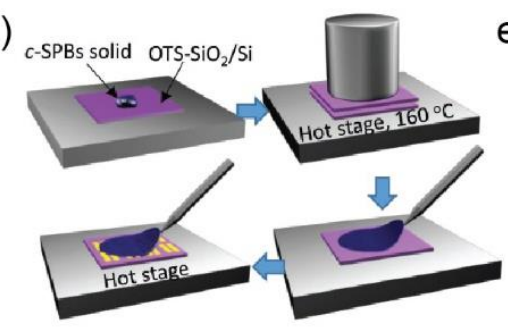

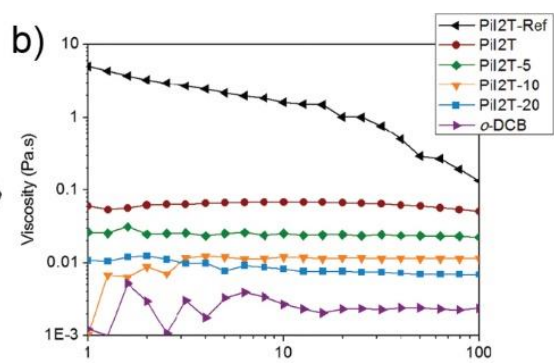

e)

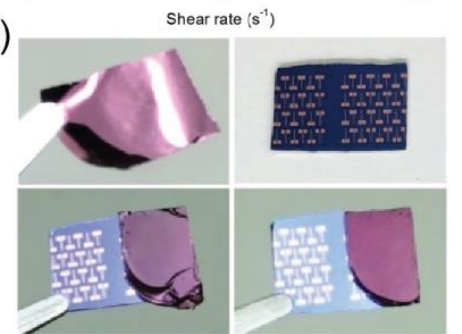

Figure 4. Panel (a) shows the chemical structure of Pil2T-X and panel (b) is the rheological flow curves of different Pil2T$X$ polymers (where $X$ is the mole percent of flexible linker) in a solution of 1,2 dicholorobenzene at $20 \mathrm{mg} \mathrm{mL}^{-1}$ at room temperature. Pil2T-Ref has different side chains see main text. Figure $4 \mathrm{~b}$ is adapted from ref. ${ }^{56}$ C $2016 \mathrm{WILEY}$-VCH Verlag GmbH \& Co. KGaA. Chemical structure of DPP-Cx shown in Scheme (d) while the schematic in panel (c) illustrates the melt-processing approach, the images in panel (f) shows the melted DPP-Cx matrix film and the corresponding devices using a peel-off and transfer approach. Figure 4d-e is adapted from ref. ${ }^{59} \Subset 2017$ WILEY-VCH Verlag GmbH \& Co. KGaA. 
Another interesting line of work leading to enhanced processability using a flexible linking strategy has been recently reported by Mei and co-workers. ${ }^{57-59}$ This group demonstrated that the inclusion of flexible linkers into the conjugated backbone of diketopyrrolopyrrole (DPP)-based semiconducting polymer coded DPP-Cx (see chemical structure in Figure 4c) had a profound influence on its melting transition, and that the blending of a polymer containing non-conjugated linkers, DPP-C5, in a matrix with its fully-conjugated counterpart, DPP-C0, that the flexible linker functions as a tie-chain which improves charge transport. ${ }^{57,58}$ The blending of the two polymers was shown to impart strong intermolecular interactions between the components that stabilize its morphology, and even permitted the melt processing of the polymers. In general, melt processing (which involves a reversible liquefaction-solidification process and is widely used in industry to produce commodity plastic thin films ${ }^{89}$ ) is a promising approach to afford the solvent-free "green" manufacturing of organic electronic devices. Zhao et al. demonstrated melt processing with the DPP-Cx system using an extrusion process (see schematic Figure 4d) where a continuous film was obtained by hot-pressing the polymer blend sandwiched between two octadecyltrichlorosilane modified $\mathrm{SiO}_{2} / \mathrm{Si}$ wafers on a hot plate. The polymer film was then peeled off and transferred onto a pre-patterned TFT substrate. Photographs of the resulting continuous polymer films and the transfer process are shown in Figure 4e. Furthermore, the meltprocessed TFTs obtained an excellent average $\mu_{\mathrm{h}}$ of $0.4 \mathrm{~cm}^{2} \mathrm{~V}^{-1} \mathrm{~S}^{-1.59}$

In addition to lowering viscosity and enabling melt processing, the flexible linker approach has recently been shown to enhance the elasticity of the resulting semiconducting polymer films. A unique functionalized FLlinker design concept presented by Bao and co-workers was found to induce an INTRINSIC stretchability and self-healing properties. ${ }^{61}$ The DPP-based conjugated polymer coded PX (see chemical structure in Figure 5a) includes a non-conjugated linker with a chemical moiety 2,3-pyridine dicarboxamide (PDCA), which was included to promote dynamic non-covalent hydrogen bond crosslinking in the polymer network (due to the presence of moderate hydrogen-bonding between the amide groups as illustrated in the schematic in Figure 5b). The authors proposed that the non-covalent crosslinking moieties dissipate energy during stretching though the breaking/reforming of hydrogen bonds when strain is applied. TFTs fabricated from these materials exhibited $\mu_{\mathrm{h}}$ up to $1.3 \mathrm{~cm}^{2} \mathrm{~V}^{-1} \mathrm{~s}^{-1}$. The charge carrier mobility remained as high as $1.12 \mathrm{~cm}^{2} \mathrm{~V}^{-1} \mathrm{~s}^{-1}$ 
even at $100 \%$ strain. The effect of incorporating the non-conjugated PDCA linker on the TFT performance is clear. When comparing the $\mu_{\mathrm{h}}$ under strain of the control polymer P1 (with 0 mol\% of the PDCA linker) the mobility is seen to decrease significantly with applied strain (See Figure 5c,d). However, when P3 (with 10 mol\% PDCA was used) the $\mu_{\mathrm{h}}$ (measured in the direction parallel or perpendicular to the applied strain) was less affected. Moreover, $\mu_{\mathrm{h}}$ of damaged devices could be almost fully recovered using a solvent/thermal healing treatment.
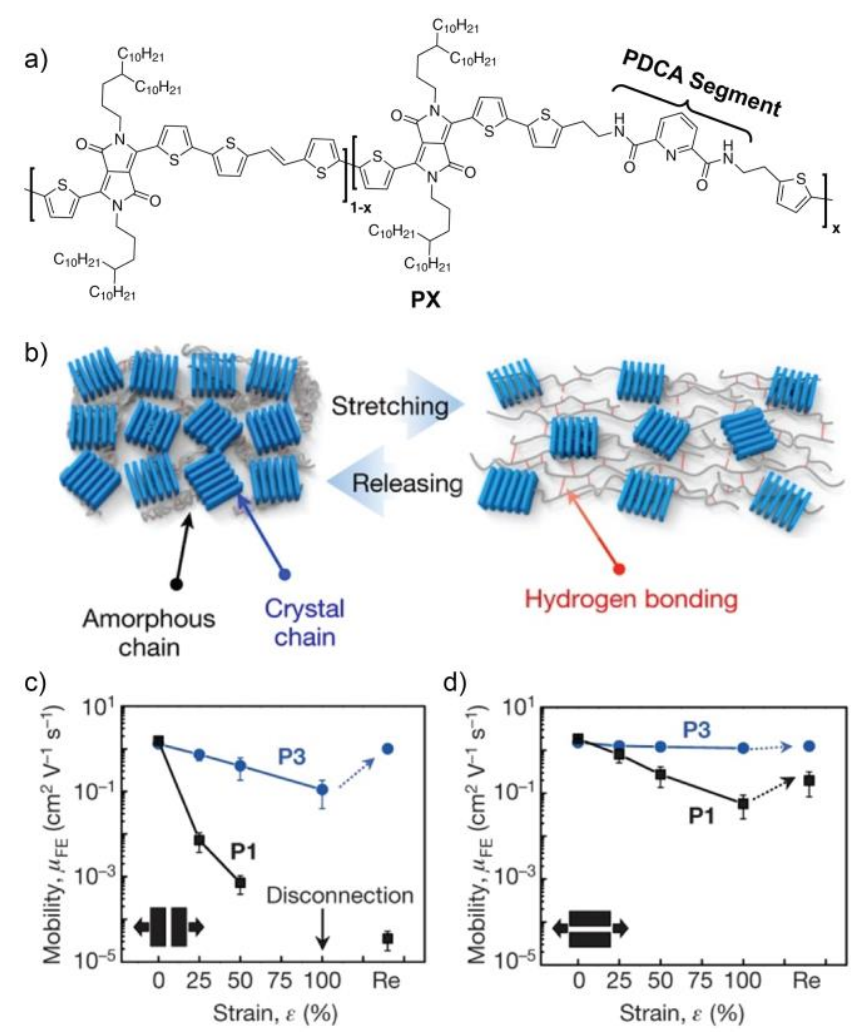

Figure 5. Enhancing elasticity with a non-conjugated linker. Panel (a) shows the chemical structure of PX. The proposed general mechanism for the enhancement of stretchability in the polymer via dynamic bonding illustrated in the schematic in panel (b). Field-effect (hole) mobility $\mu_{\mathrm{FE}}$ as a function of various strains is shown for P1 (0 mol\% PDCA linker) and P3 (10 mol\% PDCA linker) along the strain direction (c) and perpendicular to strain direction (d). The point "Re" corresponds to the measurement after relaxing the film. Figure 5b-c is adapted from ref (C) 2016 Macmillan Publishers Limited, part of Springer Nature.

In another example, Savagatrup et al. used copolymers of the aforementioned DPP-Cx $(x=3)$ with a fully conjugated monomer and measured the crack-onset strain. ${ }^{90}$ They found that with increasing fraction of flexible monomer the crack onset strain increased, suggesting more elastic properties with the flexible linker incorporation. Interestingly, despite the authors initial hypothesis that the pure DPP-C3 (with fully flexible backbone) would be the most ductile, they observed this material to have the greatest brittleness. It was suggested that the smallest lamellar spacing and also the greatest lamellar order present in that material 
lead to the surprizing deviation. This reinforces the idea that both the molecular structure and the packing arrangement in the solid state govern the mechanical (and of course optoelectronic) properties of the resulting material. Overall these initial results demonstrating the tuning elasticity of semiconducting polymers represent a major step forward towards skin-inspired stretchable organic electronics that could be integrated into wearable devices.

\section{Conclusion}

The examples highlighted in this focus review have illustrated that introducing non-conjugated break spacers (i.e. flexible linkers) between $\pi$-conjugated segments in polymer semiconductors is a promising tool to engineer the self-assembly of these materials while preserving the core electronic nature of the $\pi$-conjugated backbone. It was shown that charge transport can be relatively preserved despite the presence of aliphatic spacers between $\pi$-conjugated segments, this has given new insight into the roles of inter- and intramolecular charge transport in these materials. The flexible linker approach has also been broadly applied to control the morphology of polymer semiconductors. In addition, this approach proved useful to improving the connectivity of small molecule organic semiconductor thin films when used as a tie-in polymer additive in order to stabilize the active layer morphology. Moreover, the ability of this approach to ease the rigidity of the conjugated polymer backbone has afforded progress toward developing alternative processing techniques (e.g. tuning of viscosity of the polymer for solution shearing depositions, and permitting the solvent-free melt-processing of polymer thin films). Furthermore, functionalizing the non-conjugated aliphatic spacer to promote hydrogen bonding has shown unique self-assembly motifs that permitted stretchability and self-healing properties. While there remains much to explore in this field, like the precise effect of the linker length and position on the self-assembly, the flexible linker approach shows much promise. Future efforts in this field will likely improve our ability at controlling morphology and also open up vast possibilities to employ alternative processing techniques such as "green" and scalable melt processing or introducing functional properties for flexible devices especially for bioelectronics and wearable, stretchable devices. ${ }^{91}$ 


\section{Acknowledgements}

The authors thank the European Research Commission (ERC starting grant "CEMOS" Project 336506) and the Swiss National Science Foundation (200021_169215) for financial support.

\section{References}

1 Thejo Kalyani, N. \& Dhoble, S. J. Organic light emitting diodes: Energy saving lighting technology-A review. Renew. Sust. Energy Rev. 16, 2696-2723, (2012).

2 Sirringhaus, H. 25th Anniversary Article: Organic Field-Effect Transistors: The Path Beyond Amorphous Silicon. Adv. Mater. 26, 1319-1335, (2014).

3 Kang, H., Kim, G., Kim, J., Kwon, S., Kim, H. \& Lee, K. Bulk-Heterojunction Organic Solar Cells: Five Core Technologies for Their Commercialization. Adv. Mater. 28, 7821-7861, (2016).

4 Someya, T., Bao, Z. \& Malliaras, G. G. The rise of plastic bioelectronics. Nature 540, 379-385, (2016).

5 Morin, P.-O., Bura, T. \& Leclerc, M. Realizing the full potential of conjugated polymers: innovation in polymer synthesis. Mater. Horiz. 3, 11-20, (2016).

Beaujuge, P. M. \& Fréchet, J. M. J. Molecular Design and Ordering Effects in $\pi$-Functional Materials for Transistor and Solar Cell Applications. J. Am. Chem. Soc. 133, 20009-20029, (2011).

Anthony, J. E. Organic electronics: Addressing challenges. Nat. Mater. 13, 773-775, (2014).

Street, R. A. Unraveling Charge Transport in Conjugated Polymers. Science 341, 1072-1073, (2013). characterization of the basic parameters governing charge transport. Proc. Natl. Acad. Sci. U.S.A. 99, 5804-5809, (2002).

Zhan, C.-G., Nichols, J. A. \& Dixon, D. A. Ionization Potential, Electron Affinity, Electronegativity, Hardness, and Electron Excitation Energy: Molecular Properties from Density Functional Theory Orbital Energies. J. Phys. Chem. A 107, 4184-4195, (2003).

11 Oberhofer, H., Reuter, K. \& Blumberger, J. Charge Transport in Molecular Materials: An Assessment of Computational Methods. Chem. Rev. 117, 10319-10357, (2017).

12 Northrup, J. E. Atomic and electronic structure of polymer organic semiconductors: P3HT, PQT, and PBTTT. Phys. Rev. B 76, 245202, (2007).

13 Hiszpanski, A. M., Khlyabich, P. P. \& Loo, Y.-L. Tuning kinetic competitions to traverse the rich structural space of organic semiconductor thin films. MRS Commun. 5, 407-421, (2015). 

R., Gerlach, A., Milita, S., Schreiber, F. \& Biscarini, F. Molecular Reorganization in Organic Field-Effect Transistors and Its Effect on Two-Dimensional Charge Transport Pathways. ACS Nano 7, 1257-1264, (2013).

Noriega, R., Rivnay, J., Vandewal, K., Koch, F. P. V., Stingelin, N., Smith, P., Toney, M. F. \& Salleo, A. A general relationship between disorder, aggregation and charge transport in conjugated polymers. Nat. Mater. 12, 1038-1044, (2013).

17 Galindo, S., Tamayo, A., Leonardi, F. \& Mas-Torrent, M. Control of Polymorphism and Morphology in Solution Sheared Organic Field-Effect Transistors. Adv. Funct. Mater. 27, 1700526, (2017).

Jones, A. O. F., Chattopadhyay, B., Geerts, Y. H. \& Resel, R. Substrate-Induced and Thin-Film Phases: Polymorphism of Organic Materials on Surfaces. Adv. Funct. Mater. 26, 2233-2255, (2016).

Diao, Y., Lenn, K. M., Lee, W.-Y., Blood-Forsythe, M. A., Xu, J., Mao, Y., Kim, Y., Reinspach, J. A., Park, S., Aspuru-Guzik, A., Xue, G., Clancy, P., Bao, Z. \& Mannsfeld, S. C. B. Understanding Polymorphism in Organic Semiconductor Thin Films through Nanoconfinement. J. Am. Chem. Soc. 136, 1704617057, (2014).

Rivnay, J., Jimison, L. H., Northrup, J. E., Toney, M. F., Noriega, R., Lu, S., Marks, T. J., Facchetti, A. \& Salleo, A. Large modulation of carrier transport by grain-boundary molecular packing and microstructure in organic thin films. Nat. Mater. 8, 952-958, (2009).

Proctor, C. M., Kher, A. S., Love, J. A., Huang, Y., Sharenko, A., Bazan, G. C. \& Nguyen, T.-Q. Understanding Charge Transport in Molecular Blend Films in Terms of Structural Order and Connectivity of Conductive Pathways. Adv. Energy Mater. 6, 1502285, (2016).

Lee, S. S., Muralidharan, S., Woll, A. R., Loth, M. A., Li, Z., Anthony, J. E., Haataja, M. \& Loo, Y.-L. Understanding Heterogeneous Nucleation in Binary, Solution-Processed, Organic Semiconductor Thin Films. Chem. Mater. 24, 2920-2928, (2012). Wang, C.-L. Packing Principles for Donor-Acceptor Oligomers from Analysis of Single Crystals. Chem. Mater. 28, 5175-5190, (2016). Morphology-Performance Relationships in Organic Solar Cells: A Protocol Review. Chem. Mater. 29, 346-354, (2016).

Henson, Z. B., Müllen, K. \& Bazan, G. C. Design strategies for organic semiconductors beyond the molecular formula. Nat. Chem. 4, 699, (2012).

27 Brandt, J. R., Salerno, F. \& Fuchter, M. J. The added value of small-molecule chirality in technological applications. Nat. Rev. Chem. 1, 0045, (2017). 

New Photocatalysts for Polymerization Reactions. Acc. Chem. Res. 49, 1980-1989, (2016). organic semiconductor thin films. Energy Environ. Sci. 7, 2145-2159, (2014).

31 Treat, N. D., Nekuda Malik, J. A., Reid, O., Yu, L., Shuttle, C. G., Rumbles, G., Hawker, C. J., Chabinyc, M. L., Smith, P. \& Stingelin, N. Microstructure formation in molecular and polymer semiconductors assisted by nucleation agents. Nat. Mater. 12, 628-633, (2013).

32 Scaccabarozzi, A. D. \& Stingelin, N. Semiconducting:insulating polymer blends for optoelectronic applications-a review of recent advances. J. Mater. Chem. A 2, 10818-10824, (2014).

33 Hiszpanski, A. M. \& Loo, Y.-L. Directing the film structure of organic semiconductors via postdeposition processing for transistor and solar cell applications. Energy Environ. Sci 7, 592-608, (2014).

Tsao, H. N. \& Mullen, K. Improving polymer transistor performance via morphology control. Chem. Soc. Rev. 39, 2372-2386, (2010).

Pisula, W., Kastler, M., Wasserfallen, D., Mondeshki, M., Piris, J., Schnell, I. \& Müllen, K. Relation between Supramolecular Order and Charge Carrier Mobility of Branched Alkyl Hexa-perihexabenzocoronenes. Chem. Mater. 18, 3634-3640, (2006).

Zhang, L., Rose, B. D., Liu, Y., Nahid, M. M., Gann, E., Ly, J., Zhao, W., Rosa, S. J., Russell, T. P., Facchetti, A., McNei, C. R., Bredas, J. L. \& Briseno, A. L. Efficient Naphthalenediimide-Based Hole Semiconducting Polymer with Vinylene Linkers between Donor and Acceptor Units. Chem. Mater. 28, 8580-8590, (2016).

37 Marszalek, T., Li, M. \& Pisula, W. Design directed self-assembly of donor-acceptor polymers. Chem. Commun. 52, 10938-10947, (2016).

38 Lee, J., Kalin, A. J., Yuan, T., Al-Hashimi, M. \& Fang, L. Fully conjugated ladder polymers. Chem. Sci. 8, 2503-2521, (2017).

39 Huang, H., Yang, L., Facchetti, A. \& Marks, T. J. Organic and Polymeric Semiconductors Enhanced by Noncovalent Conformational Locks. Chem. Rev. 117, 10291-10318, (2017).

40 Lee, T.-H., Wu, K.-Y., Lin, T.-Y., Wu, J.-S., Wang, C.-L. \& Hsu, C.-S. Role of the Comonomeric Units in Reaching Linear Backbone, High Solid-State Order and Charge Mobilities in Heptacyclic Arene-Based Alternating Copolymers. Macromolecules 46, 7687-7695, (2013).

Mei, J. \& Bao, Z. Side Chain Engineering in Solution-Processable Conjugated Polymers. Chem. Mater. 26, 604-615, (2014).

Balakrishnan, K., Datar, A., Naddo, T., Huang, J., Oitker, R., Yen, M., Zhao, J. \& Zang, L. Effect of SideChain Substituents on Self-Assembly of Perylene Diimide Molecules: Morphology Control. J. Am. Chem. Soc. 128, 7390-7398, (2006). 

W. E., Kreouzis, T., Toney, M. F. \& Bao, Z. Taming Charge Transport in Semiconducting Polymers with Branched Alkyl Side Chains. Adv. Funct. Mater. 27, 1701973, (2017).

Kang, I., Yun, H.-J., Chung, D. S., Kwon, S.-K. \& Kim, Y.-H. Record High Hole Mobility in Polymer Semiconductors via Side-Chain Engineering. J. Am. Chem. Soc. 135, 14896-14899, (2013).

45 Yao, J., Yu, C., Liu, Z., Luo, H., Yang, Y., Zhang, G. \& Zhang, D. Significant Improvement of Semiconducting Performance of the Diketopyrrolopyrrole-Quaterthiophene Conjugated Polymer through Side-Chain Engineering via Hydrogen-Bonding. J. Am. Chem. Soc. 138, 173-185, (2016).

Kang, B., Kim, R., Lee, S. B., Kwon, S.-K., Kim, Y.-H. \& Cho, K. Side-Chain-Induced Rigid Backbone Organization of Polymer Semiconductors through Semifluoroalkyl Side Chains. J. Am. Chem. Soc. 138, 3679-3686, (2016).

Tsao, H. N., Cho, D. M., Park, I., Hansen, M. R., Mavrinskiy, A., Yoon, D. Y., Graf, R., Pisula, W., Spiess, H. W. \& Müllen, K. Ultrahigh Mobility in Polymer Field-Effect Transistors by Design. J. Am. Chem. Soc. 133, 2605-2612, (2011).

Schroeder, B. C., Li, Z., Brady, M. A., Faria, G. C., Ashraf, R. S., Takacs, C. J., Cowart, J. S., Duong, D. T., Chiu, K. H., Tan, C.-H., Cabral, J. T., Salleo, A., Chabinyc, M. L., Durrant, J. R. \& McCulloch, I. Enhancing Fullerene-Based Solar Cell Lifetimes by Addition of a Fullerene Dumbbell. Angew. Chem. Int. Ed. 53, 12870-12875, (2014).

Gasperini, A., Jeanbourquin, X. A., Rahmanudin, A., Yu, X. \& Sivula, K. Enhancing the Thermal Stability of Solution-Processed Small-Molecule Semiconductor Thin Films Using a Flexible Linker Approach. Adv. Mater. 27, 5541-5546, (2015).

Liang, Z., Cormier, R. A., Nardes, A. M. \& Gregg, B. A. Developing perylene diimide based acceptor polymers for organic photovoltaics. Synth. Met. 161, 1014-1021, (2011).

Xiang, X., Shao, W., Liang, L., Chen, X.-Q., Zhao, F.-G., Lu, Z., Wang, W., Li, J. \& Li, W.-S. Photovoltaic poly(rod-coil) polymers based on benzodithiophene-centred A-D-A type conjugated segments and dicarboxylate-linked alkyl non-conjugated segments. RSC Adv. 6, 23300-23309, (2016). Microwire Growth and Crystal Packing of $\pi$-Moieties. Chem. Mater. 24, 1944-1949, (2012). Saeki, A., Seki, S. \& Yagai, S. Covalent Modular Approach for Dimension-Controlled Self-Organization of Perylene Bisimide Dyes. Chem. Eur. J. 19, 6561-6565, (2013). transport with a flexible-linker approach. Chem. Sci. 5, 4922-4927, (2014). 

Conjugated Flexible Linkers in Semiconducting Polymers: A Pathway to Improved Processability without Compromising Device Performance. Adv. Electron. Mater. 2, 1600104, (2016). Polymers: Impact on Polymer Processability and Charge Transport Properties. Macromolecules 48, 2048-2053, (2015). Semiconducting Polymer Blends: The Influence of Conjugation-Break Spacer Length in Matrix Polymers. Macromolecules 49, 2601-2608, (2016).

Zhao, Y., Zhao, X., Roders, M., Gumyusenge, A., Ayzner, A. L. \& Mei, J. Melt-Processing of Complementary Semiconducting Polymer Blends for High Performance Organic Transistors. Adv. Mater. 29, 1605056, (2017).

Erdmann, T., Fabiano, S., Milián-Medina, B., Hanifi, D., Chen, Z., Berggren, M., Gierschner, J., Salleo, A., Kiriy, A., Voit, B. \& Facchetti, A. Naphthalenediimide Polymers with Finely Tuned In-Chain $\pi-$ Conjugation: Electronic Structure, Film Microstructure, and Charge Transport Properties. Adv. Mater. 28, 9169-9174, (2016).

61 Oh, J. Y., Rondeau-Gagné, S., Chiu, Y.-C., Chortos, A., Lissel, F., Wang, G.-J. N., Schroeder, B. C., Kurosawa, T., Lopez, J., Katsumata, T., Xu, J., Zhu, C., Gu, X., Bae, W.-G., Kim, Y., Jin, L., Chung, J. W., Tok, J. B. H. \& Bao, Z. Intrinsically stretchable and healable semiconducting polymer for organic transistors. Nature 539, 411, (2016).

Noël, C. \& Navard, P. Liquid crystal polymers. Prog. Polym. Sci. 16, 55-110, (1991).

Chung, T. S. The recent developments of thermotropic liquid crystalline polymers. Polym. Eng. Sci. 26, 901-919, (1986).

Sokolik, I., Yang, Z., Karasz, F. E. \& Morton, D. C. Blue-light electroluminescence from p-phenylene vinylene-based copolymers. J. Appl. Phys. 74, 3584-3586, (1993).

Padmanaban, G. \& Ramakrishnan, S. Conjugation Length Control in Soluble Poly[2-methoxy-5-((2'ethylhexyl)oxy)-1,4-phenylenevinylene] (MEHPPV): Synthesis, Optical Properties, and Energy Transfer. J. Am. Chem. Soc. 122, 2244-2251, (2000).

Martens, H. C. F., Blom, P. W. M. \& Schoo, H. F. M. Comparative study of hole transport in poly(pphenylene vinylene) derivatives. Phys. Rev. B 61, 7489-7493, (2000).

67 Hu, D., Yu, J., Padmanaban, G., Ramakrishnan, S. \& Barbara, P. F. Spatial Confinement of Exciton Transfer and the Role of Conformational Order in Organic Nanoparticles. Nano Lett. 2, 1121-1124, (2002). Polymers Using Defect Inclusion Strategies. J. Am. Chem. Soc. 133, 10155-10160, (2011). 

Oligo(phenylenevinylene)s and Flexible Chains. Macromolecules 45, 5051-5057, (2012).

Traub, M. C., DuBay, K. H., Ingle, S. E., Zhu, X., Plunkett, K. N., Reichman, D. R. \& Vanden Bout, D. A. Chromophore-Controlled Self-Assembly of Highly Ordered Polymer Nanostructures. J. Phys. Chem. Lett. 4, 2520-2524, (2013).

71 Gasperini, A. \& Sivula, K. Effects of Molecular Weight on Microstructure and Carrier Transport in a Semicrystalline Poly(thieno)thiophene. Macromolecules 46, 9349-9358, (2013).

Zhang, X., Bronstein, H., Kronemeijer, A. J., Smith, J., Kim, Y., Kline, R. J., Richter, L. J., Anthopoulos, T. D., Sirringhaus, H., Song, K., Heeney, M., Zhang, W., McCulloch, I. \& DeLongchamp, D. M. Molecular origin of high field-effect mobility in an indacenodithiophene-benzothiadiazole copolymer. Nat. Commun. 4, 2238, (2013).

Sciascia, C., Martino, N., Schuettfort, T., Watts, B., Grancini, G., Antognazza, M. R., Zavelani-Rossi, M., McNeill, C. R. \& Caironi, M. Sub-Micrometer Charge Modulation Microscopy of a High Mobility Polymeric n-Channel Field-Effect Transistor. Adv. Mater. 23, 5086-5090, (2011).

Kronemeijer, A. J., Gili, E., Shahid, M., Rivnay, J., Salleo, A., Heeney, M. \& Sirringhaus, H. A Selenophene-Based Low-Bandgap Donor-Acceptor Polymer Leading to Fast Ambipolar Logic. Adv. Mater. 24, 1558-1565, (2012).

Beiley, Z. M., Hoke, E. T., Noriega, R., Dacuña, J., Burkhard, G. F., Bartelt, J. A., Salleo, A., Toney, M. F. \& McGehee, M. D. Morphology-Dependent Trap Formation in High Performance Polymer Bulk Heterojunction Solar Cells. Adv. Energy Mater. 1, 954-962, (2011).

Steyrleuthner, R., Schubert, M., Howard, I., Klaumünzer, B., Schilling, K., Chen, Z., Saalfrank, P., Laquai, F., Facchetti, A. \& Neher, D. Aggregation in a High-Mobility n-Type Low-Bandgap Copolymer with Implications on Semicrystalline Morphology. J. Am. Chem. Soc. 134, 18303-18317, (2012).

Pasveer, W. F., Cottaar, J., Tanase, C., Coehoorn, R., Bobbert, P. A., Blom, P. W. M., de Leeuw, D. M. \& Michels, M. A. J. Unified Description of Charge-Carrier Mobilities in Disordered Semiconducting Polymers. Phys. Rev. Lett. 94, 206601, (2005). Processed Small Molecule Solar Cells. Adv. Energy Mater. 7, 1602242-n/a, (2017). polymers having alternative conjugated and non-conjugated segments. Chem. Commun. 50, 77207722, (2014). Sivula, K. Engineering the self-assembly of diketopyrrolopyrrole-based molecular semiconductors via an aliphatic linker strategy. J. Mater. Chem. A 5, 10526-10536, (2017). 
Fullerene-Based Solar Cell Lifetimes by Addition of a Fullerene Dumbbell. Angew. Chem. Int. Ed. 53, 12870-12875, (2014).

82 Lin, X., Hirono, M., Seki, T., Kurata, H., Karatsu, T., Kitamura, A., Kuzuhara, D., Yamada, H., Ohba, T., Saeki, A., Seki, S. \& Yagai, S. Covalent Modular Approach for Dimension-Controlled Self-Organization of Perylene Bisimide Dyes. Chem. Eur. J. 19, 6561-6565, (2013).

83 Lee, D. H., Lee, J. H., Kim, H. J., Choi, S., Park, G. E., Cho, M. J. \& Choi, D. H. (D)n-[sigma]-(A)m type partially conjugated block copolymer and its performance in single-component polymer solar cells. J. Mater. Chem. A 5, 9745-9751, (2017).

84 Lombeck, F., Sepe, A., Thomann, R., Friend, R. H. \& Sommer, M. Compatibilization of All-Conjugated Polymer Blends for Organic Photovoltaics. ACS Nano 10, 8087-8096, (2016).

85 Lombeck, F., Komber, H., Sepe, A., Friend, R. H. \& Sommer, M. Enhancing Phase Separation and Photovoltaic Performance of All-Conjugated Donor-Acceptor Block Copolymers with Semifluorinated Alkyl Side Chains. Macromolecules 48, 7851-7860, (2015).

86 Rahmanudin, A., Jeanbourquin, X. A., Hänni, S., Sekar, A., Ripaud, E., Yao, L. \& Sivula, K. Morphology stabilization strategies for small-molecule bulk heterojunction photovoltaics. J. Mater. Chem. A 5, 17517-17524, (2017).

87 Koch, F. P. V., Rivnay, J., Foster, S., Müller, C., Downing, J. M., Buchaca-Domingo, E., Westacott, P., Yu, L., Yuan, M., Baklar, M., Fei, Z., Luscombe, C., McLachlan, M. A., Heeney, M., Rumbles, G., Silva, C., Salleo, A., Nelson, J., Smith, P. \& Stingelin, N. The impact of molecular weight on microstructure and charge transport in semicrystalline polymer semiconductors-poly(3-hexylthiophene), a model study. Prog. Polym. Sci. 38, 1978-1989, (2013).

88 Zhao, K., Khan, H. U., Li, R., Su, Y. \& Amassian, A. Entanglement of Conjugated Polymer Chains Influences Molecular Self-Assembly and Carrier Transport. Adv. Funct. Mater. 23, 6024-6035, (2013).

89 John M Dealy, J. W. Melt Rheology and its Applications in the Plastics Industry. 2 edn, (Springer Netherlands, 2013).

90 Savagatrup, S., Zhao, X., Chan, E., Mei, J. \& Lipomi, D. J. Effect of Broken Conjugation on the Stretchability of Semiconducting Polymers. Macromol. Rapid Commun. 37, 1623-1628, (2016).

91 Wang, G. J. N., Gasperini, A. \& Bao, Z. Stretchable Polymer Semiconductors for Plastic Electronics. Adv. Electron. Mater. 4, 1700429, (2018). 
